\title{
Interhospital referral of colorectal cancer patients: a Dutch population-based study
}

\author{
A. K. Warps ${ }^{1,2}$ - M. P. M. de Neree tot Babberich ${ }^{3}$ - E. Dekker ${ }^{3}$ - M. W. J. M. Wouters ${ }^{1,2,4}$ • J. W. T. Dekker ${ }^{5}$. \\ R. A. E. M. Tollenaar ${ }^{1,2} \cdot$ P. J. Tanis $^{6}(1) \cdot$ On behalf of the Dutch ColoRectal Audit
}

Accepted: 4 February 2021 / Published online: 20 March 2021

(C) The Author(s) 2021

\begin{abstract}
Purpose Interhospital referral is a consequence of centralization of complex oncological care but might negatively impact waiting time, a quality indicator in the Netherlands. This study aims to evaluate characteristics and waiting times of patients with primary colorectal cancer who are referred between hospitals.

Methods Data were extracted from the Dutch ColoRectal Audit (2015-2019). Waiting time between first tumor-positive biopsy until first treatment was compared between subgroups stratified for referral status, disease stage, and type of hospital.

Results In total, 46,561 patients were included. Patients treated for colon or rectal cancer in secondary care hospitals were referred in $12.2 \%$ and $14.7 \%$, respectively. In tertiary care hospitals, corresponding referral rates were $43.8 \%$ and $66.4 \%$. Referred patients in tertiary care hospitals were younger, but had a more advanced disease stage, and underwent more often multivisceral resection and simultaneous metastasectomy than non-referred patients in secondary care hospitals $(p<0.001)$. Referred patients were more often treated within national quality standards for waiting time compared to non-referred patients $(p<0.001)$. For referred patients, longer waiting times prior to MDT were observed compared to non-referred patients within each hospital type, although most time was spent post-MDT.

Conclusion A large proportion of colorectal cancer patients that are treated in tertiary care hospitals are referred from another hospital but mostly treated within standards for waiting time. These patients are younger but often have a more advanced disease. This suggests that these patients are willing to travel more but also reflects successful centralization of complex oncological patients in the Netherlands.
\end{abstract}

Keywords Interhospital referral $\cdot$ Centralization $\cdot$ Waiting time $\cdot$ Colorectal cancer care

P. J. Tanis

p.j.tanis@amsterdamumc.nl

1 Department of Surgery and Biomedical Data Sciences, Leiden University Medical Center, Albinusdreef 2,

2333ZA Leiden, Netherlands

2 Scientific Bureau, Dutch Institute for Clinical Auditing, Rijnsburgerweg 10, 2333AA Leiden, Netherlands

3 Department of Gastroenterology and Hepatology, Amsterdam University Medical Centers, University of Amsterdam, Meibergdreef 9, 1105AZ Amsterdam, Netherlands

4 Department of Surgical Oncology, Antoni van Leeuwenhoek Hospital, Plesmanlaan 121, 1066CX Amsterdam, Netherlands

5 Department of Surgery, Reinier de Graaf Groep, Reinier de Graafweg 5, 2625AD Delft, Netherlands

6 Department of Surgery, Cancer Centre Amsterdam, Amsterdam University Medical Centers, University of Amsterdam, Meibergdreef 9, 1105AZ Amsterdam, Netherlands

\section{Introduction}

Waiting times in oncologic care may cause psychological harm to patients [1-5]. The underlying concern relates to potential progression of the tumor during the diagnostic work-up and waiting time until start of first treatment [6-9]. In the Netherlands, this has led to the development of quality standards for waiting times, set up by the Dutch Federation of Oncologic Specialties (SONCOS) [10]. For colorectal cancer, a maximum waiting time from diagnosis until first treatment of 5 weeks was established. If a patient is referred to another hospital, an extra 3 weeks can be added to the maximum waiting time. Although no profound scientific evidence exists for the association of delays in colorectal cancer treatment on survival [11-15], which could be due to the slow tumor progression $[16,17]$, it is increasingly seen as a hospital quality indicator. 
Waiting times remain a challenge for hospitals [18], and the ongoing centralization of complex surgery to high-volume centers [19-21], causes an increasing burden on hospital resources $[19,22]$. Although most of the waiting time seems to take place in the (pre-) diagnostic phase [23-25], time from diagnosis until treatment has the most impact on patient satisfaction [25].

In the Dutch healthcare system, cancer networks organize weekly consultation of regional hospitals by a tertiary hospital. Minimal hospital volumes and defining complexity of disease that needs referral to an expert center are the driving forces in interhospital referral. There is, however, minimal insight in redistribution of patients through interhospital referral and the impact on waiting times and subsequent outcomes. Therefore, this study aimed to determine the characteristics of patients referred between hospitals in the Netherlands, with the corresponding waiting times compared to patients who were not referred. For this purpose, hospitals were stratified as secondary and tertiary care hospitals.

\section{Methods}

Data were derived from the Dutch ColoRectal Audit (DCRA), a disease-specific national audit. This audit is mandatory and registers patients, tumor, treatment, and short-term outcome characteristics of all patients undergoing resection for primary colorectal cancer in the Netherlands [26]. Referral status was included in the audit since January 2015.

Hospitals were categorized into secondary or tertiary care hospitals, based on specialization in the treatment of colorectal cancer and the availability of advanced treatment modalities. Of the 75 hospitals in the Netherlands in 2015-2019, eight university hospitals, one cancer center, and one referral center for locally advanced cancer were classified as tertiary care hospitals. Further information about the distribution and characteristics of Dutch hospitals have been described earlier [27].

\section{Patients}

For this study, no ethical approval or informed consent was required under Dutch law. All patients $(n=52,666)$ who underwent resection between January 1, 2015, and December 31, 2019, were evaluated. Minimal data requirements were tumor location, date of surgery, and 30-day/inhospital mortality. In addition, only patients operated in an elective setting without synchronous tumors $(n=46,572)$, and with information on the date of first tumor-positive biopsy and date of operation were selected, resulting in a total eligible number of 46,561 patients.

\section{Data extraction and outcome parameters}

The following data were extracted from the DCRA database: patient and disease characteristics, procedural characteristics, and 30-day or in-hospital postoperative outcome.

For the evaluation of waiting times, the following dates were used from the DCRA: first tumor-positive biopsy, first multidisciplinary team meeting (MDT), and first treatment given to the patient (neoadjuvant (preoperative radiotherapy or systemic treatment), metastasectomy, or resection of the primary tumor).

Because of its potential implications for waiting times in secondary and tertiary care hospitals, patients were categorized into three groups: group (1) non-locally advanced/nonmetastatic colon or rectal cancer (cT1-3N0-2M0); group (2) locally advanced/non-metastatic (cT4N0-2M0); and group (3) metastatic colon or rectal cancer (M1). If clinical stage was missing and no neoadjuvant treatment was given, pathological stage was used.

To assess possible delay in the diagnostic phase, time between first positive biopsy and MDT was calculated and categorized as $0-2,3-4,5-6$, and $\geq 7$ weeks. The date of the MDT was not registered in 2018 , so only patients that underwent surgery between 2015 and 2017 or in 2019 were included for this specific analysis. To evaluate total waiting time, time from first tumor-positive biopsy until first treatment was calculated and categorized as $\leq 5$ weeks, 6 to 8 weeks, and $>8$ weeks, according to Dutch quality standards.

\section{Data analysis}

Analyses were performed separately for colon and rectal cancer and for secondary and tertiary care hospitals. Patients were classified as non-referred (NRF) and referred (RF) based on registered referral status in the DCRA. Risk factors for a waiting time from first tumor-positive biopsy until treatment of more than 5 weeks were determined using multivariable logistic analysis. Multicollinearity was tested with the variance inflation factor (VIF). A VIF of $>2.5$ was considered as multicollinearity and resulted in removing one of the factors. Differences in waiting times were analyzed with a KruskalWallis rank-sum test for continuous variables or Chi-square test for categorical variables. A $p$-value of less than 0.05 was considered statistically significant. All analyses were performed in R Studio version 3.6.1 (2020).

\section{Results}

\section{Baseline characteristics and neoadjuvant treatment}

A total of 31,560 patients with colon cancer and 15,001 with rectal cancer were included for analysis. Table 1 shows the 


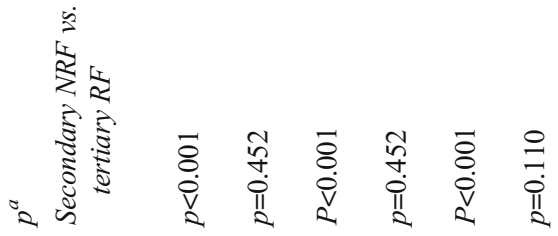

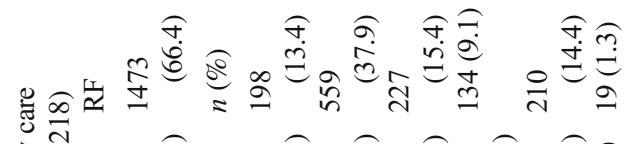

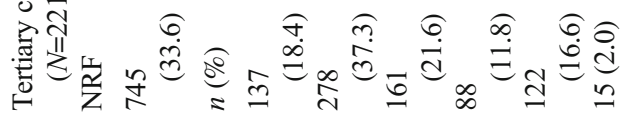

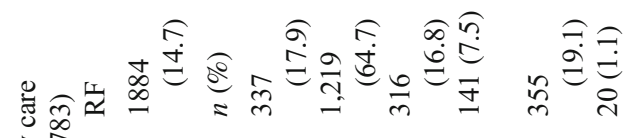

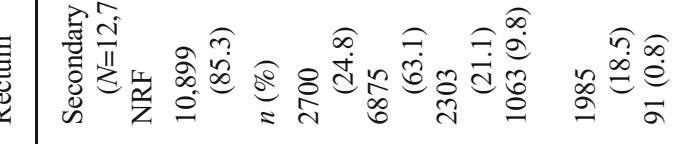

它

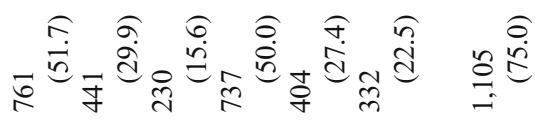

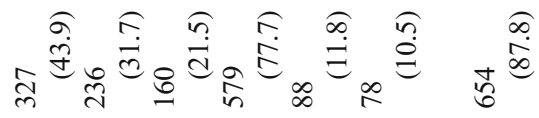

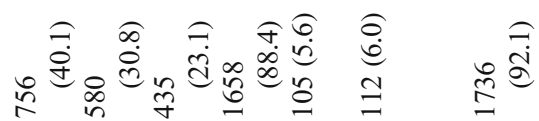

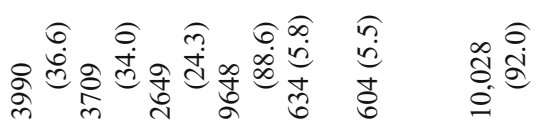

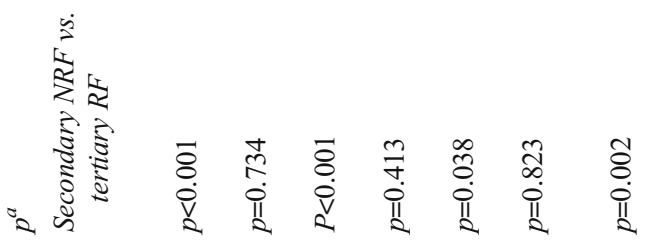

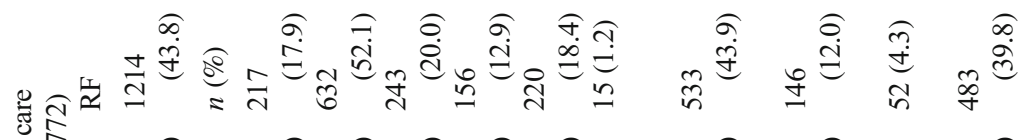

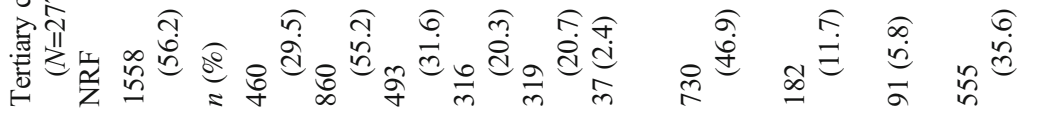

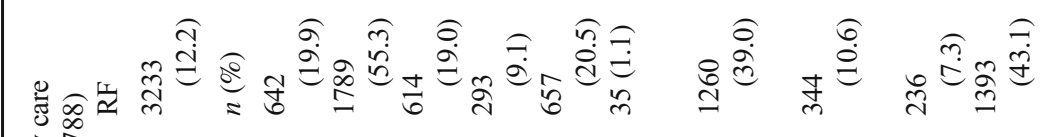

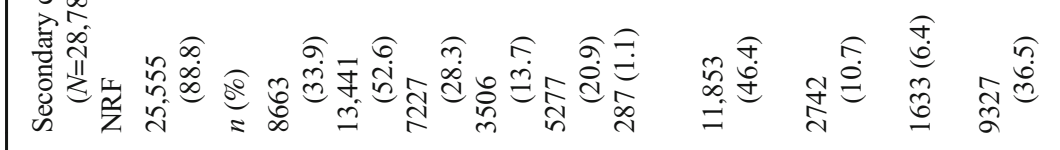

官

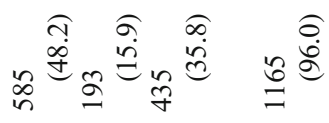

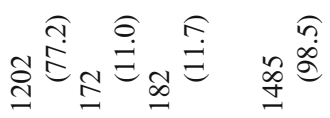

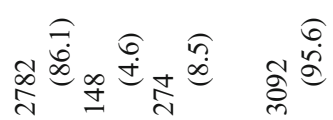

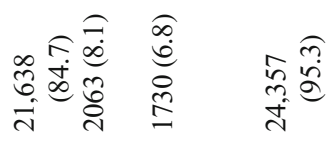


characteristics of the NRF and RF patients per type of hospital (secondary and tertiary) for colon and rectum separately. In secondary care hospitals, $12.2 \%$ of the patients with colon cancer and $14.7 \%$ of the patients with rectal cancer were referred from another hospital. In tertiary care hospitals, this was $43.8 \%$ and $66.4 \%$, respectively. Similar baseline differences between RF patients in tertiary care hospitals to NRF patients in secondary care hospitals were found for colon cancer and rectal cancer. RF patients were less often $\geq 75$ years, had less often an ASA 3+ score, had less frequently a BMI of 30+, had a higher T-stage, had more often metastasis (M1), had undergone more often a surgical intervention preceding the resection, and received more often neoadjuvant treatment. In addition, RF rectal cancer patients in tertiary care hospitals had more often a tumor located $\leq 5 \mathrm{~cm}$ of the anal verge.

\section{Patient, tumor, and neoadjuvant treatment characteristics associated with referral to tertiary care hospitals}

In colon cancer patients, a significant higher odds for referral to a tertiary care hospital was found for locally advanced and metastatic disease (AOR 2.514, 95\% CI 2.074-3.046 for group 2 (T4N0-2M0) and AOR 5.560, 95\% CI 4.740-6.522 for group 3 (M1)), if a stoma was created for obstruction (AOR 2.010, 95\% CI 1.522-2.656) and when neo-adjuvant chemotherapy was given (AOR 6.231, 95\% CI 4.989-7.783). Significant lower odds were observed for age $75+($ AOR $0.535,95 \%$ CI 0.454-0.631), ASA III+ (AOR 0.726, 95\% CI 0.615-0.856), and when the primary tumor was located in the descending colon (AOR 0.532, 95\% CI 0.381-0.743) (Table 2).

In rectal cancer, significant higher odds for referral to a tertiary hospital was found for previous abdominal surgery (AOR 2.374, 95\% CI 1.357-4.156), tumor location ( $\leq 5 \mathrm{~cm}$ from anal verge; AOR 1.881, 95\% CI 1.578-2.244; 6-10cm AOR 1.242, 95\% CI 1.301-1.497), locally advanced and metastatic tumors (AOR 5.839, 95\% CI 4.938-6.909 for group 2 and AOR 5.071, 95\% CI 4.226-6.085 for group 3), construction of a stoma preceding the resection (AOR $1.968,95 \% \mathrm{CI}$ 1.639-2.362), and neoadjuvant therapy (AOR $1.458,95 \%$ CI 1.259-1.686 for chemotherapy and AOR $1.462,95 \%$ CI $1.248-1.712$ for radiotherapy). In contrast, a significant lower odds ratio was seen for age 75+ (AOR $0.516,95 \%$ CI 0.433 0.616), ASA III + (AOR 0.692, 95\% CI 0.680-0.967), and BMI 30+ (AOR 0.811, 95\% CI 0.680-0.967).

\section{Waiting time}

After excluding unknown $\mathrm{T}$ or $\mathrm{M}$ stage and inconclusive endoscopic biopsy, a total of 30,615 patients with colon cancer and 14,039 with rectal cancer were evaluable for analyzing waiting times. Figure 1 shows the median waiting times, with their corresponding interquartile range (IQR) from first tumor- 
positive biopsy until first treatment for secondary and tertiary care hospitals, stratified for the different patient groups.

Within each group, all RF colon and rectal cancer patients in tertiary care hospitals had a significantly longer waiting time compared to NRF patients in secondary care hospitals. Mean waiting time differences ranged from 5 days (group 3 rectal cancer, 40 vs. 35 days, $p<0.001$ ) to 17 days (group 3 colon cancer, 42 vs. 25 days, $p<0.001$ ).

Within secondary care hospitals, waiting time was longer for RF compared to NRF patients in all colon cancer subgroups, with waiting time differences ranging from 3 (group 1,31 vs. 28 days, $p<0.001$ ) to 12 days (group 3,37 vs. 25 days, $p=0.001$ ). Referred patients that were treated in secondary care hospitals waited longer than NRF patients in case of non-locally advanced/non-metastasized disease (group 1, 36 vs. 33 days, $p<0.001$ ) and metastasized disease (group 3, 42 vs. 35 days, $p=0.003$ ).

Among all colon cancer subgroups in tertiary care hospitals, RF patients waited significantly longer than NRF patients, ranging from 6 (group 3, 42 vs. 36 days, $p<0.001$ ) to 13 days (group 2, 40 vs. 27 days, $p<0.001$ ). For rectal cancer treated in tertiary centers, these waiting time differences ranged from 6 (group 1, 41 vs. 35 days, $p<0.001$ ) to 9 days (group 3,40 vs. 31 days, $p=0.006$ ).

\section{Adherence to quality standards}

Supplementary table 1 shows that for colon cancer patients in secondary care hospitals, the overall waiting time was $\leq 5$ weeks in $73.6 \%$ for NRF patients and $\leq 8$ weeks in $89.2 \%$ for RF patients $(p<0.001)$. Corresponding percentages in tertiary care hospitals were $57.1 \%$ and $70.4 \%(p<0.001)$, respectively.

For rectal cancer treatment in secondary care hospitals, $58.3 \%$ of the NRF patients were treated $\leq 5$ weeks, and $82.7 \%$ of the RF patients were treated $\leq 8$ weeks $(p<0.001)$. In tertiary hospitals, these percentages were $53.9 \%$ and $76.3 \%$, respectively $(p<0.001)$. Supplementary figure 1 gives

Table 2 Factors related with referral

\begin{tabular}{|c|c|c|c|}
\hline & & Colon & Rectum \\
\hline & & Adjusted odds ratio $(95 \% \mathrm{CI})$ & Adjusted odds ratio $(95 \% \mathrm{CI})$ \\
\hline \multirow[t]{2}{*}{ Age } & $<75$ years & Ref. & Ref. \\
\hline & $\geq 75$ years & $0.535(0.454-0.631)$ & $0.516(0.433-0.616)$ \\
\hline \multirow[t]{2}{*}{ ASA score } & I-II & Ref. & Ref. \\
\hline & III+ & $0.726(0.615-0.856)$ & $0.692(0.581-0.824)$ \\
\hline \multirow[t]{2}{*}{ BMI (kg/m2) } & $\leq 30$ & Ref. & Ref. \\
\hline & $>30$ & $1.045(0.886-1.232)$ & $0.811(0.680-0.967)$ \\
\hline \multirow[t]{2}{*}{ Previous abdominal surgery } & No & Ref. & Ref. \\
\hline & Yes & $1.195(0.621-2.299)$ & $2.374(1.357-4.156)$ \\
\hline \multirow[t]{4}{*}{ Location of tumor } & Ascending colon (incl. hepatic flexure) & Ref. & \\
\hline & Transverse colon (incl. splenic flexure) & $1.099(0.890-1.351)$ & \\
\hline & Descending colon & $0.532(0.381-0.743)$ & \\
\hline & Sigmoid colon & $0.921(0.797-1.066)$ & \\
\hline \multirow[t]{3}{*}{ Tumor distance from anal verge } & $>10 \mathrm{~cm}$ & & Ref. \\
\hline & $6-10 \mathrm{~cm}$ & & $1.242(1.301-1.497)$ \\
\hline & $\leq 5 \mathrm{~cm}$ & & $1.881(1.578-2.244)$ \\
\hline \multirow[t]{3}{*}{ Tumor group } & 1. (T1-3N0-2M0) & Ref. & Ref. \\
\hline & 2. (T4N0-2M0) & $2.514(2.074-3.046)$ & $5.839(4.938-6.909)$ \\
\hline & 3. (M1) & $5.560(4.740-6.522)$ & $5.071(4.226-6.085)$ \\
\hline \multirow[t]{2}{*}{ Surgical intervention preceding the resection } & No & Ref. & Ref. \\
\hline & Stoma & $2.010(1.522-2.656)$ & $1.968(1.639-2.362)$ \\
\hline \multirow[t]{2}{*}{ Neo adjuvant chemotherapy } & No & Ref. & Ref. \\
\hline & Yes & $6.231(4.989-7.783)$ & $1.458(1.259-1.686)$ \\
\hline \multirow[t]{2}{*}{ Neoadjuvant radiotherapy } & No & & Ref. \\
\hline & Yes & & $1.462(1.248-1.712)$ \\
\hline Modelfit: Nagelkerke $R^{2}$ : & & 0.167 & 0.222 \\
\hline
\end{tabular}

Multivariable logistic regression assessing patient, tumor, and neo-adjuvant factors associated with referral to tertiary hospitals compared to non-referred patient in secondary care hospitals 


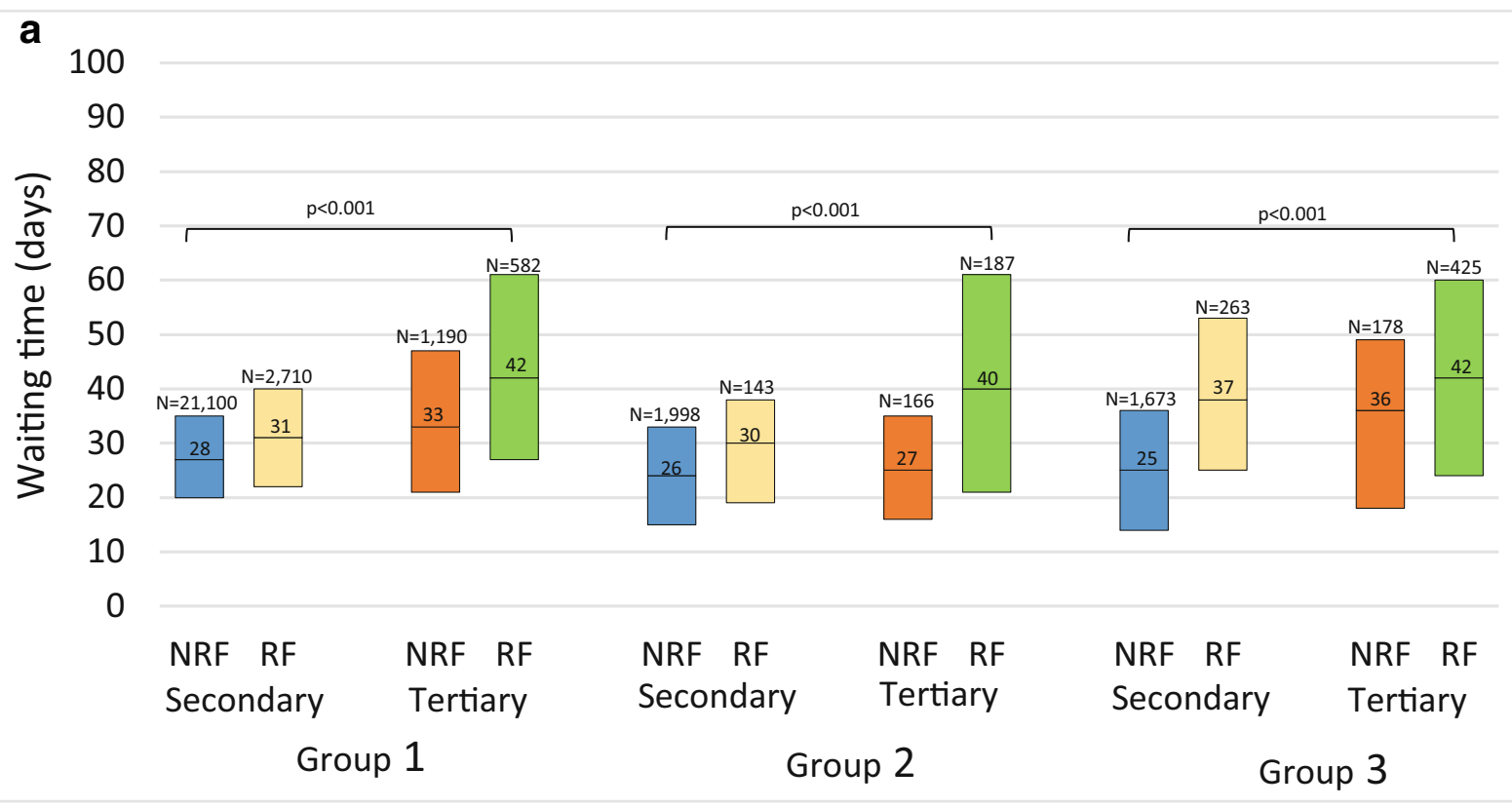

\section{b}

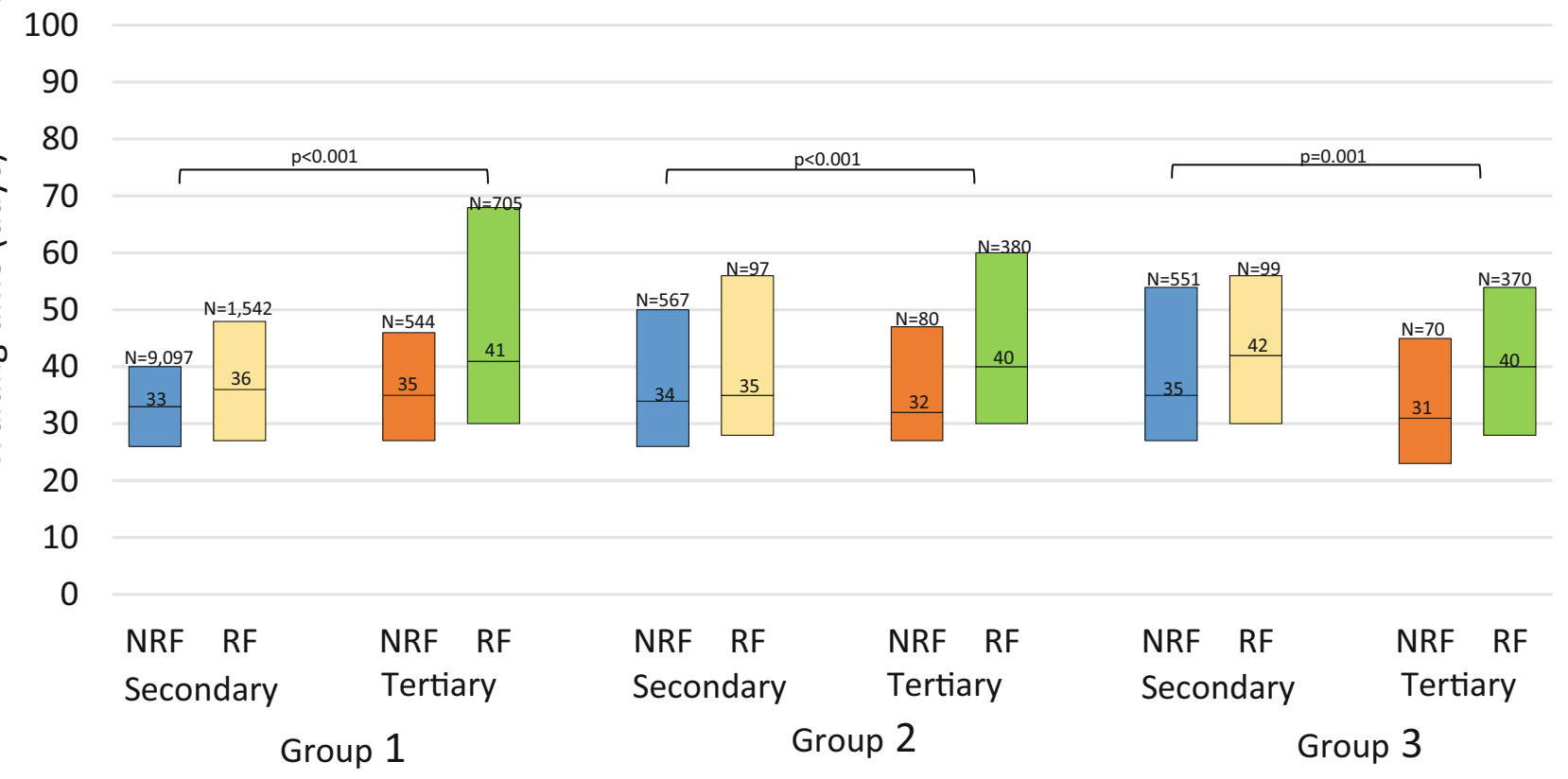

Fig. 1 a Waiting times in days for colon cancer patients. Figure 1 a shows the waiting time (days) for NRF and RF colon cancer patients in secondary and tertiary hospitals. A Chi-square test was used. Group 1, T1-3N02M0; group 2, T4N0-2M0; group3, M1. b Waiting times in days for

an overview of median waiting times per hospital between first tumor positive biopsy and first treatment for secondary and tertiary care hospitals.

\section{Diagnostic and referral delay}

The date of multidisciplinary team meeting (MDT) was registered in 20,229 colon and 9954 rectal cancer patients. Time from first tumor-positive biopsy until MDT was significantly rectal cancer patients. Figure $1 \mathrm{~b}$ shows the waiting time (days) for $\mathrm{NRF}$ and RF rectal cancer patients in secondary and tertiary hospitals. A Chi-square test was used. Group 1, T1-3N0-2M0; group 2, T4N0-2M0; group3, M1

longer for RF colon cancer patients compared to NRF patients in both secondary $(p<0.001)$ and tertiary care hospitals $(p<0.001)$ as shown in Fig. 2. This was also found for rectal cancer in both types of hospitals. The time from first MDT until first treatment was significantly longer for RF colon cancer patients compared to NRF patients, but with minimal absolute differences. In contrast, the time from first MDT until first treatment did not significantly differ between RF and NRF rectal cancer patients. 


\section{Surgical characteristics}

Table 3 shows that RF patients in tertiary care hospitals differed significantly for the majority of surgical characteristics compared with NRF patients in secondary hospitals. RF colon and rectal cancer patients in tertiary care hospitals had a higher risk of getting a stoma ( $p<0.001$ and $p<0.001$, respectively), had more often an additional resection due to tumor invasion $(p<0.001$ and $p<0.001$, respectively), and had more often a simultaneous resection for metastasis $(p<0.001$ and $p<0.001$, respectively) compared to NRF patients in secondary hospitals.

\section{Discussion}

This population-based study shows that waiting times for referred colorectal cancer patients differ substantially from those of patients treated in their hospital of diagnosis. For both secondary and tertiary care hospitals, it is hard to meet the current Dutch waiting time standards in every single case, but remarkably this seems more difficult in NRF patients compared to RF patients. According to Dutch quality standards, waiting times for the latter patient population may be 3 weeks longer (eight versus five weeks), which explains this remarkable observation. A large proportion of patients treated in tertiary care hospitals consist of referred patients with advanced disease, both locally advanced as well as metastatic. The complexity of these patients also translates into the type of treatments given. For example, more diverting stomas were constructed in these patients with more often neoadjuvant treatment. From these observations, we conclude that centralization of more complex patients has been successful in the Netherlands over the past years, with efficient referral to tertiary care hospitals. For RF patients, longer waiting times mainly occur during diagnostic or referral phase (pre-MDT). Once patients have been discussed in the MDT, RF colon cancer patients experience only a minimal longer waiting time.

Waiting times are always of concern for patients and healthcare providers. Identifying and understanding factors associated with longer waiting times are essential information when discussing waiting time regulations. Simunovic et al. found that age was associated with treatment delay for breast, colon, lung, and prostate cancer [28]. Furthermore, Bilimoria et al. found that with several nonmetastatic cancers (breast, colon, esophageal, gastric, liver, pancreatic, and rectal), besides age, also comorbidities (charslon2+), stage 1 disease (compared to stage 3), or treatment at a national cancer institute was associated with a treatment delay $>30$ days [18]. We found that RF patients had longer waiting times compared to NRF patients. We also found age, ASA III+, tumor stage, tumor location (part of the colon (colon cancer) or distance to the anal verge (rectal cancer)), and neoadjuvant treatment to be associated with RF. One might hypothesize that this reflects adequate prioritization by the surgeons. On the other hand, the RF population is a heterogeneous population that differs not only regarding tumor characteristics but also comprises patients with severe comorbidities that require specific perioperative care as well as relatively young, healthy, and highly educated patients that are more willing to travel for treatment. This demonstrates that centralization of more complex patients has been successful in the Netherlands over the past years, with efficient referral to tertiary care hospitals.

Nevertheless, we demonstrated that a substantial number of hospitals do not meet the waiting time standards and a significant
Fig. 2 Waiting times for biopsy until MDT and MDT until treatment. Figure 2 shows the waiting times (weeks) for biopsy until MDT and MDT until treatment for NRF and RF patient in secondary and tertiary hospitals

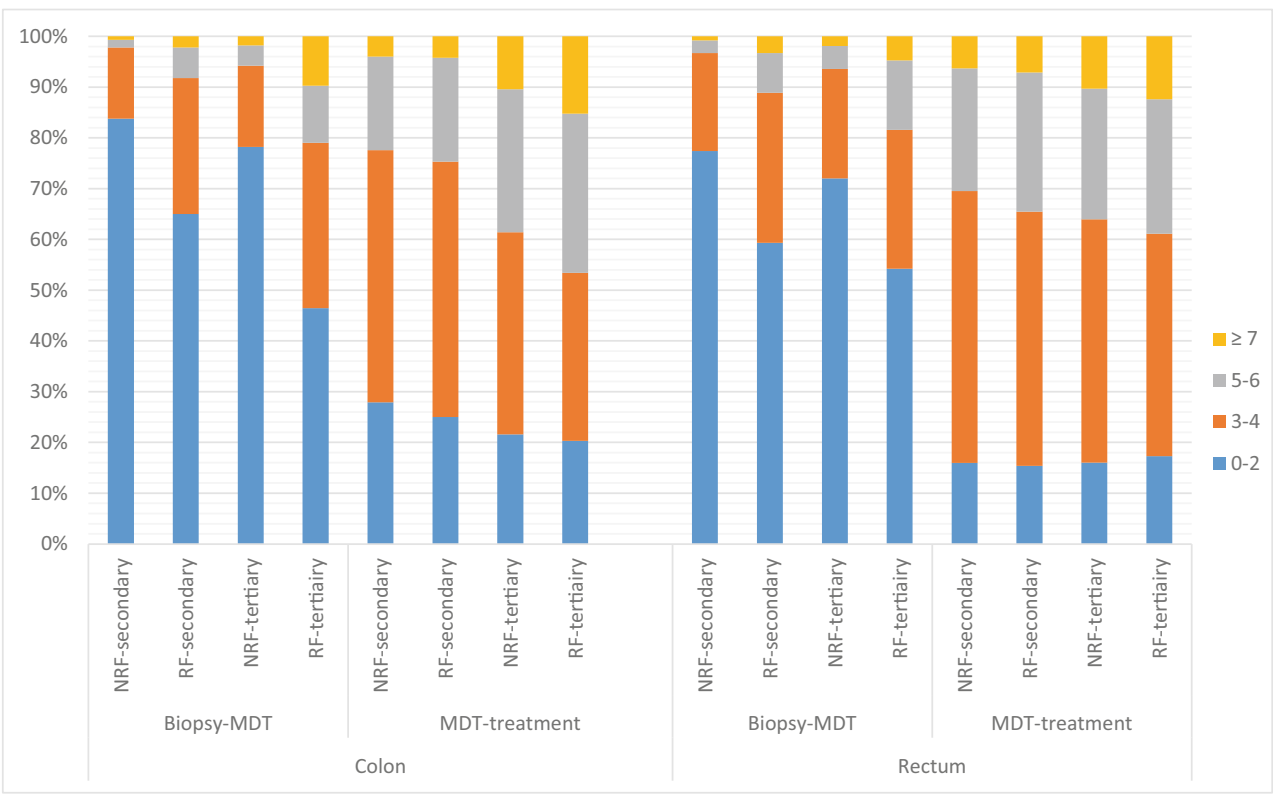




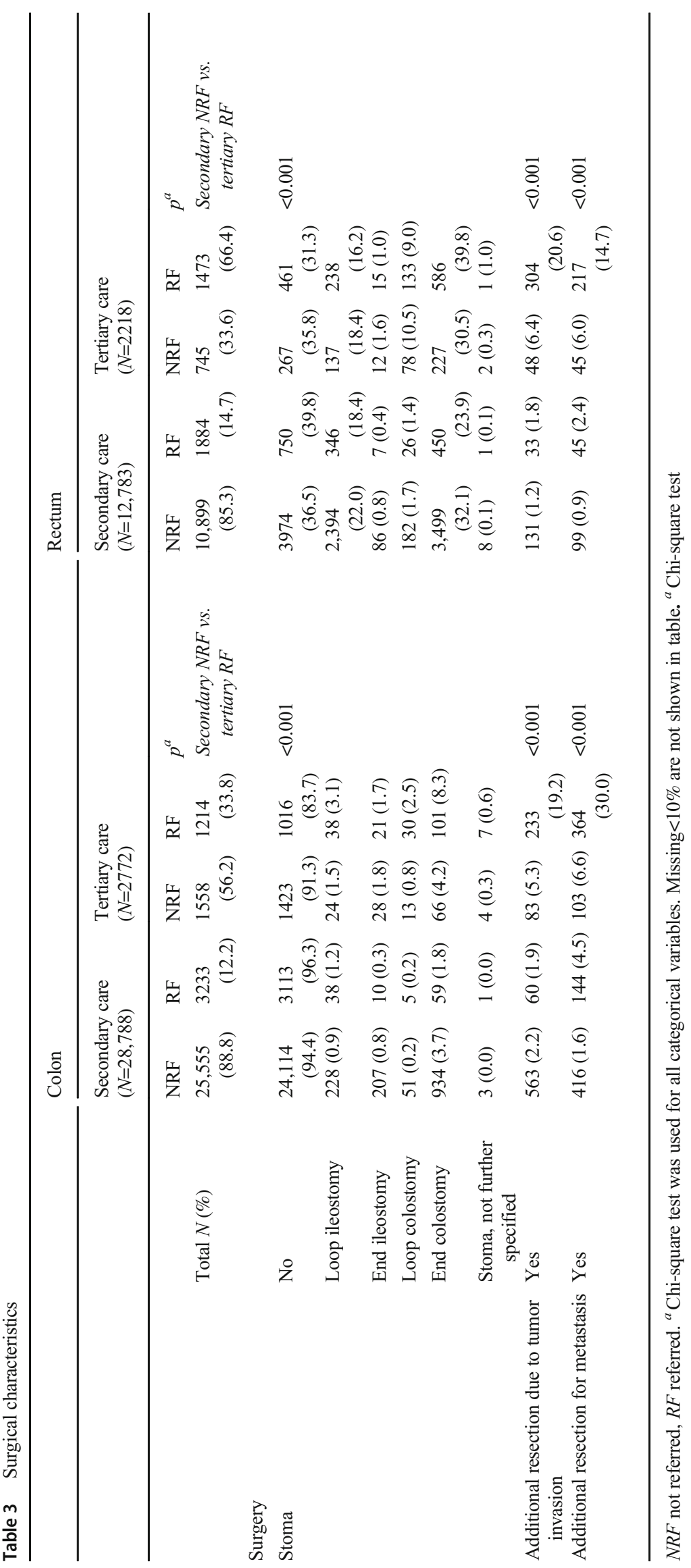


impact of interhospital referral on waiting time was found. Centralization of complex care to tertiary care hospitals is important to pursue, but should ideally not cause delay in treatment. Tertiary care centers are not necessarily high-volume centers, and the majority of less complex care is probably best concentrated in high-volume secondary centers in an efficient manner. In the Netherlands, we are working on a potential solution and strategy to achieve this. By regionalization and echeloning of care with regional MDT meetings, waiting times can potentially be reduced with optimal centralization of complex care. Discussion of patients early in the cancer care process with good triage and exchange of patients between hospitals and willingness to refer are key elements. This will result in the referral of highly complex care to tertiary centers but also the exchange of low-complex patients to peripheral high-volume hospitals. By creating regional networks with a central portal where patients come in, efficient distribution of the "right patient to the right place" is aimed for.

Although no analysis of postoperative outcomes was performed, other studies did not find an association between delay in treatment and worse outcomes. Porter et al. showed that most of the delay was caused in the (pre) diagnostic phase. So, from the perspective of tumor biology, interventions should be aimed at improving access to these medical resources [25]. Bevis et al. found a significant delay until consultation by a specialist or initiation of treatment for patients referred through a non-fast track route, but this delay was not associated with a more advanced stage of disease or a reduction in potentially curative operations [13]. Also, the studies of Murchie et al. [29] and Helewa et al. [30] did not find a relationship between provider's delay and stage at diagnosis or survival of colorectal cancer. In 2000, the UK introduced the 2-week rule to reduce diagnostic delay, which has not improved 2-year survival [31], and also meeting waiting time criteria showed no improvement in 1-year survival [32]. A systematic review that addressed the literature between 2008 and 2017 for treatment delay in colon cancer treatment found no association between delay and a reduced disease-free survival, 5-years survival, and overall survival for patients with colon cancer [33]. However, literature remains contradictory. For example, a systematic review of 34 studies (2000-2020) on 17 therapy indications by Hanna et al. found a consistent and significant increase in mortality for each 4 weeks delay of cancer surgery, with a hazard ratio of 1.06 (95\% CI 1.01-1.12) for colectomy [34], and Lee showed that the risk of death was increased for patients with a waiting time of more than 30 days between diagnosis and treatment [35], whereas Strous et al. and Trepanier et al. showed that treatment exceeding a month did not decrease overall and recurrence-free survival [36, 37]. Amri et al. did not find worse cancer outcomes for treatment delay in colon cancer and even found an inverse relation between treatment delay and mortality and recurrence rates [38].
Even though most studies demonstrated that no (or at most a controversial) relationship between waiting time until treatment of colorectal cancer and worse outcomes exist, this should not be a reason to stop putting effort in reducing waiting times. It should be regarded as an indication that waiting times within reasonable ranges do not harm the patient from tumor biology perspective. However, psychological distress of the patients should also be taken into account. In a systematic review, Brocken et al. found that a rapid diagnostic pathway may be beneficial for the distress levels of patients eventually not having cancer, but this association was not seen in patients finally receiving the diagnosis of cancer [39]. In our study, the most significant differences in waiting time for RF compared to NRF patients were found in the time period before MDT. During this time interval, the additional diagnostic work-up and actual referral take place. Besides the fact that the referral process will take a certain amount of time, it is most likely that more complex patients also have a more extensive (diagnostic) work-up prior to the MDT, supporting the MDT in the discussion conducted for the most appropriate treatment options. To a certain extent, the additional diagnostic work-up and referral process run parallel to each other, making it challenging to quantify the actual proportion of waiting time that can be attributed solely to referral. For the pre-treatment phase, the need for a more comprehensive, personalized based approach may be beneficial, like Song et al. showed in a study on waiting times for the treatment of gastric and esophageal cancer [40]. Besides, extra waiting time could be a conscious choice, for example, to perform a more profound work-up, and does not necessarily have to be negative. Increased waiting time might also include patient delay of any reason (e.g., psychologically adapting to the diagnosis). Some patients have specific preferences for a hospital or surgeon. Like McConnell et al. discussed, the relationship between waiting times in colorectal cancer care and quality of care is complex [41], and multiple perspectives need to be taken into account.

The strength of the present study is the large number of patients and external validity related to the population-based data reflecting daily practice. However, some limitations need to be addressed. A certain degree of missing data is inevitable in population-based studies. Considering case-mix adjustment, there is always a possibility that not all contributing factors were included. Besides, we did not have any detailed information on patient, referral, or diagnostic delays (e.g., until colonoscopy). This prevents us from profoundly understanding the buildup of waiting times. Furthermore, no information on referral source (the type of hospital) was available. So, referral from secondary to tertiary care hospitals and the other way around could not be quantified or analyzed. Finally, the DCRA does not provide information on disease-free survival and overall (long-term) survival, which might be of relevance in the discussion on interhospital referral and waiting times. 
In conclusion, this population-based study showed that a substantial number of hospitals do not meet current waiting time standards, and interhospital referrals had a significant impact on waiting time prior to the MDT. Referred colorectal cancer patients appeared to be younger healthier that have more advanced disease, in need of more complex treatment. The Dutch system seems to be able to efficiently concentrate low-volume high-complex disease. However, centralization of complex care seemed to be accompanied by an increased waiting time, and effort need to be made to prevent this since the impact of delay on survival is controversial. Regional networks, with efficient patient distribution based on the complexity of care needed, might prevent longer waiting times in tertiary care hospitals.

Availability of data and material The data that support the findings of this study are available from the Dutch Institute for Clinical Auditing (DICA) but are not publicly available. Data are however available from the authors upon reasonable request and with permission of the Dutch Institute for Clinical Auditing and the Dutch ColoRectal Audit Board.

Code availability Statistical codes that are used for this study is available from the authors upon reasonable request and with permission of the Dutch Institute

Supplementary Information The online version contains supplementary material available at https://doi.org/10.1007/s00384-021-03881-2.

\section{Declarations}

Ethics approval and consent to participate Obligatory audit from the inspectorate of healthcare which required no informed consent from patients for data collection. Data analyses were performed on a anonymized dataset and does not need ethical approval according to Dutch law.

\section{Consent for publication Not applicable.}

Competing interests The authors declare no competing interests.

Open Access This article is licensed under a Creative Commons Attribution 4.0 International License, which permits use, sharing, adaptation, distribution and reproduction in any medium or format, as long as you give appropriate credit to the original author(s) and the source, provide a link to the Creative Commons licence, and indicate if changes were made. The images or other third party material in this article are included in the article's Creative Commons licence, unless indicated otherwise in a credit line to the material. If material is not included in the article's Creative Commons licence and your intended use is not permitted by statutory regulation or exceeds the permitted use, you will need to obtain permission directly from the copyright holder. To view a copy of this licence, visit http://creativecommons.org/licenses/by/4.0/.

\section{References}

1. Risberg T, Sørbye SW, Norum J, Wist EA (1996) Diagnostic delay causes more psychological distress in female than in male cancer patients. Anticancer Research 16(2):995-1000

2. Zabora J, Brintzenhofeszoc K, Curbow B et al (2001) The prevalence of psychological distress by cancer site. Psycho-Oncology 10(1):19-28. https://doi.org/10.1002/1099-1611(200101/02)10: $1<19::$ AID-PON501>3.0.CO;2-6

3. Keller M, Sommerfeldt S, Fischer C, Knight L, Riesbeck M, Löwe B, Herfarth C, Lehnert T (2004) Recognition of distress and psychiatric morbidity in cancer patients: a multi-method approach. Annals of Oncology 15(8):1243-1249. https://doi.org/10.1093/ annonc/mdh 318

4. Ford S, Lewis S, Fallowfield L (1995) Psychological morbidity in newly referred patients with cancer. Journal of Psychosomatic Research 39(2):193-202. https://doi.org/10.1016/0022-3999(94) 00103-C

5. Gray RE, Fitch MI, Phillips C, Labrecque M, Klotz L (1999) Presurgery experiences of prostate cancer patients and their spouses. Cancer Practice 7(3):130-135. https://doi.org/10.1046/j. 1523-5394.1999.07308.x

6. Yun YH, Kim YA, Min YH, Park S, Won YJ, Kim DY, Choi IJ, Kim YW, Park SJ, Kim JH, Lee DH, Yoon SJ, Jeong SY, Noh DY, Heo DS (2012) The influence of hospital volume and surgical treatment delay on long-term survival after cancer surgery. Annals of Oncology 23(10):2731-2737. https://doi.org/10.1093/annonc/ mds 101

7. Richards MA, Westcombe AM, Love SB, Littlejohns P, Ramirez AJ (1999) Influence of delay on survival in patients with breast cancer: a systematic review. Lancet 353(9159):1119-1126. https://doi.org/10.1016/S0140-6736(99)02143-1

8. Van Harten MC, Hoebers FJP, Kross KW et al (2015) Determinants of treatment waiting times for head and neck cancer in the Netherlands and their relation to survival. Oral Oncology 51(3): 272-278. https://doi.org/10.1016/j.oraloncology.2014.12.003

9. Elit LM, O'Leary EM, Pond GR, Seow HY (2014) Impact of wait times on survival for women with uterine cancer. Journal of Clinical Oncology 32(1):27-33. https://doi.org/10.1200/JCO.2013.51.3671

10. (SONCOS) DF of OS www.soncos.org

11. Simunovic M, Rempel E, Thériault ME et al (2009) Influence of delays to nonemergent colon cancer surgery on operative mortality, disease-specific survival and overall survival. Canadian Journal of Surgery. https://doi.org/10.1016/S0008-428X(09)50109-9

12. Ramos M, Esteva M, Cabeza E, Campillo C, Llobera J, Aguiló A (2007) Relationship of diagnostic and therapeutic delay with survival in colorectal cancer: a review. European Journal of Cancer 43(17):2467-2478. https://doi.org/10.1016/j.ejca.2007.08.023

13. Bevis PM, Donaldson OW, Card M, Durdey P, Thomas MG, Sylvester PA, Longman RJ (2008) The association between referral source and stage of disease in patients with colorectal cancer. Colorectal Disease 10(1):58-62. https://doi.org/10.1111/j.14631318.2007.01222.x

14. Ramos M, Esteva M, Cabeza E, Llobera J, Ruiz A (2008) Lack of association between diagnostic and therapeutic delay and stage of colorectal cancer. European Journal of Cancer 44(4):510-521. https://doi.org/10.1016/j.ejca.2008.01.011

15. Zafar A, Mak T, Whinnie S, Chapman MAS (2012) The 2-week wait referral system does not improve 5-year colorectal cancer survival. Colorectal Disease. 14:e177-e180. https://doi.org/10.1111/j. 1463-1318.2011.02826.x

16. Fearon ER, Vogelstein B (1990) A genetic model for colorectal tumorigenesis. Cell 61(5):759-767. https://doi.org/10.1016/00928674(90)90186-I 
17. Manne U, Shanmugam C, Katkoori VR, Bumpers HL, Grizzle WE (2011) Development and progression of colorectal neoplasia. Cancer Biomarkers 9(1-6):235-265. https://doi.org/10.3233/ CBM-2011-0160

18. Bilimoria KY, Ko CY, Tomlinson JS, Stewart AK, Talamonti MS, Hynes DL, Winchester DP, Bentrem DJ (2011) Wait times for cancer surgery in the United States: trends and predictors of delays. Annals of Surgery 253(4):779-785. https://doi.org/10.1097/SLA. 0b013e318211cc0f

19. Prades $\mathrm{J}$, Manchon-Walsh $\mathrm{P}$, Solà $\mathrm{J}$, Espinàs JA, Guarga $\mathrm{A}$, Borras JM (2016) Improving clinical outcomes through centralization of rectal cancer surgery and clinical audit: a mixed-methods assessment. European Journal of Public Health 26(4):538-542. https:// doi.org/10.1093/eurpub/ckv237

20. Stitzenberg KB, Meropol NJ (2010) Trends in centralization of cancer surgery. Annals of Surgical Oncology 17(11):2824-2831. https://doi.org/10.1245/s10434-010-1159-0

21. Kontovounisios C, Tan E, Pawa N, Brown G, Tait D, Cunningham D, Rasheed S, Tekkis P (2017) The selection process can improve the outcome in locally advanced and recurrent colorectal cancer: activity and results of a dedicated multidisciplinary colorectal cancer centre. Colorectal Disease 19(4):331-338. https://doi.org/10. 1111/codi.13517

22. Simunovic M, Gagliardi A, McCready D, Coates A, Levine M, DePetrillo D (2001) A snapshot of waiting times for cancer surgery provided by surgeons affiliated with regional cancer centres in Ontario. Cmaj 165(4):421-425

23. Korsgaard M, Pedersen L, Laurberg S (2008) Delay of diagnosis and treatment of colorectal cancer-a population-based Danish Study. Cancer Detection and Prevention 32(1):45-51. https://doi. org/10.1016/j.cdp.2008.01.001

24. Singh H, De Coster C, Shu E et al (2010) Wait times from presentation to treatment for colorectal cancer: a population-based study. Canadian Journal of Gastroenterology 24(1):33-39. https://doi.org/ 10.1155/2010/692151

25. Porter GA, Inglis KM, Wood LA, Veugelers PJ (2005) Access to care and satisfaction in colorectal cancer patients. World Journal of Surgery 29(11):1444-1451. https://doi.org/10.1007/s00268-005$7955-1$

26. Van Leersum NJ, Snijders HS, Henneman D et al (2013) The dutch surgical colorectal audit. European Journal of Surgical Oncology 39(10):1063-1070. https://doi.org/10.1016/j.ejso.2013.05.008

27. Kolfschoten NE, Marang Van De Mheen PJ, Gooiker GA et al (2011) Variation in case-mix between hospitals treating colorectal cancer patients in the Netherlands. European Journal of Surgical Oncology 37(11):956-963. https://doi.org/10.1016/j.ejso.2011. 08.137

28. Simunovic M, Thériault ME, Paszat L et al (2005) Using administrative databases to measure waiting times for patients undergoing major cancer surgery in Ontario, 1993-2000. Canadian Journal of Surgery 48(2):137-142

29. Murchie P, Raja EA, Brewster DH, Campbell NC, Ritchie LD, Robertson R, Samuel L, Gray N, Lee AJ (2014) Time from first presentation in primary care to treatment of symptomatic colorectal cancer: effect on disease stage and survival. British Journal of Cancer 111(3):461-469. https://doi.org/10.1038/bjc.2014.352

30. Helewa RM, Turner D, Park J, Wirtzfeld D, Czaykowski P, Hochman D, Singh H, Shu E, Mckay A (2013) Longer waiting times for patients undergoing colorectal cancer surgery are not associated with decreased survival. Journal of Surgical Oncology 108(6):378-384. https://doi.org/10.1002/jso.23412

31. Walsh SR, Gilson NL, Brown K, Novell RJ (2007) Trends in colorectal cancer survival following the 2-week rule. Colorectal Disease 9 (3):207-209. https://doi.org/10.1111/j.1463-1318.2006. 01105.x

32. Di Girolamo C, Walters S, Gildea C et al (2018) Can we assess Cancer Waiting Time targets with cancer survival? A populationbased study of individually linked data from the National Cancer Waiting Times monitoring dataset in England, 2009-2013. PLoS ONE. 13:e0201288. https://doi.org/10.1371/journal.pone.0201288

33. Hangaard Hansen C, Gögenur M, Tvilling Madsen M, Gögenur I (2018) The effect of time from diagnosis to surgery on oncological outcomes in patients undergoing surgery for colon cancer: a systematic review. European Journal of Surgical Oncology 44(10): 1479-1485. https://doi.org/10.1016/j.ejso.2018.06.015

34. Hanna TP, King WD, Thibodeau S, Jalink M, Paulin GA, HarveyJones E, O'Sullivan DE, Booth CM, Sullivan R, Aggarwal A (2020) Mortality due to cancer treatment delay: systematic review and meta-analysis. BMJ (Clinical research ed) 371:m4087. https:// doi.org/10.1136/bmj.m4087

35. Lee YH, Kung PT, Wang YH, Kuo WY, Kao SL, Tsai WC (2019) Effect of length of time from diagnosis to treatment on colorectal cancer survival: a population-based study. PLoS ONE. 14: e0210465. https://doi.org/10.1371/journal.pone.0210465

36. Strous MTA, Janssen-Heijnen MLG, Vogelaar FJ (2019) Impact of therapeutic delay in colorectal cancer on overall survival and cancer recurrence - is there a safe timeframe for prehabilitation? European Journal of Surgical Oncology 45(12):2295-2301. https://doi.org/ 10.1016/j.ejso.2019.07.009

37. Trepanier M, Paradis T, Kouyoumdjian A, Dumitra T, Charlebois P, Stein BS, Liberman AS, Schwartzman K, Carli F, Fried GM, Feldman LS, Lee L (2020) The impact of delays to definitive surgical care on survival in colorectal cancer patients. Journal of Gastrointestinal Surgery 24(1):115-122. https://doi.org/10.1007/ s11605-019-04328-4

38. Amri R, Bordeianou LG, Sylla P, Berger DL (2014) Treatment delay in surgically-treated colon cancer: does it affect outcomes? Annals of Surgical Oncology 21(12):3909-3916. https://doi.org/ 10.1245/s10434-014-3800-9

39. Brocken P, Prins JB, Dekhuijzen PNR, Van Der Heijden HFM (2012) The faster the better? - A systematic review on distress in the diagnostic phase of suspected cancer, and the influence of rapid diagnostic pathways. Psycho-Oncology 21(1):1-10. https://doi.org/ 10.1002/pon.1929

40. Song H, Fang F, Valdimarsdóttir U, Lu D, Andersson TML, Hultman C, Ye W, Lundell L, Johansson J, Nilsson M, Lindblad M (2017) Waiting time for cancer treatment and mental health among patients with newly diagnosed esophageal or gastric cancer: a nationwide cohort study. BMC Cancer. 17:2. https://doi.org/10. 1186/s12885-016-3013-7

41. Mcconnell YJ, Inglis K, Porter GA (2010) Timely access and quality of care in colorectal cancer: are they related? International Journal for Quality in Health Care 22(3):219-228. https://doi.org/ 10.1093/intqhe/mzq010

Publisher's note Springer Nature remains neutral with regard to jurisdictional claims in published maps and institutional affiliations. 